\title{
THE ENTROPY OF RANDOM-FREE GRAPHONS AND PROPERTIES
}

\author{
HAMED HATAMI AND SERGUEI NORINE
}

\begin{abstract}
Every graphon defines a random graph on any given number $n$ of vertices. It was known that the graphon is random-free if and only if the entropy of this random graph is subquadratic. We prove that for random-free graphons, this entropy can grow as fast as any subquadratic function. However, if the graphon belongs to the closure of a random-free graph property, then the entropy is $O(n \log n)$. We also give a simple construction of a non-stepfunction random-free graphon for which this entropy is linear, refuting a conjecture of Janson.
\end{abstract}

\section{INTRODUCTION}

In recent years a theory of convergent sequences of dense graphs has been developed. One can construct a limit object for such a sequence in the form of certain symmetric measurable functions called graphons. Every graphon defines a random graph on any given number of vertices. In [HJS] several facts about the asymptotics of the entropies of these random variables are established. These results provide a good understanding of the situation when the graphon is not "random-free". However in the case of the random-free graphons they completely trivialize. The purpose of this article is to study these entropies in the case of the random-free graphons.

1.1. Preliminaries. For every natural number $n$, denote $[n]:=\{1, \ldots, n\}$. In this paper all graphs are simple and finite. For a graph $G$, let $V(G)$ and $E(G)$, respectively denote the set of the vertices and the edges of $G$. Let $\mathcal{U}$ denote set of all graphs up to an isomorphism. Moreover, for $n \geq 0$, let $\mathcal{U}_{n} \subset \mathcal{U}$ denote the set of all graphs in $\mathcal{U}$ with exactly $n$ vertices. We will usually work with labeled graphs. For every $n \geq 1$, denote by $\mathcal{L}_{n}$ the set of all graphs with vertex set $[n]$.

The homomorphism density of a graph $H$ in a graph $G$, denoted by $t(H ; G)$, is the probability that a random mapping $\phi: V(H) \rightarrow V(G)$ preserves adjacencies, i.e. $u v \in E(H)$ implies $\phi(u) \phi(v) \in E(G)$. The induced density of a graph $H$ in a graph $G$, denoted by $p(H ; G)$, is the probability that a random embedding of the vertices of $H$ in the vertices of $G$ is an embedding of $H$ in $G$.

We call a sequence of finite graphs $\left(G_{n}\right)_{n=1}^{\infty}$ convergent if for every finite graph $H$, the sequence $\left\{p\left(H ; G_{n}\right)\right\}_{n=1}^{\infty}$ converges. It is not difficult to construct convergent sequences $\left(G_{n}\right)_{n=1}^{\infty}$ such that their limits cannot be recognized as graphs, i.e. there is no graph $G$, with $\lim _{n \rightarrow \infty} p\left(H ; G_{n}\right)=p(H ; G)$ for every $H$. Thus naturally one considers $\overline{\mathcal{U}}$, the completion of $\mathcal{U}$ under this notion of convergence. It is not hard to see that $\overline{\mathcal{U}}$ is a compact metrizable space which contains $\mathcal{U}$ as a dense subset. The elements of the complement $\mathcal{U}^{\infty}:=\overline{\mathcal{U}} \backslash \mathcal{U}$ are called graph limits. Note that a sequence of graphs $\left(G_{n}\right)_{n=1}^{\infty}$ converges to a graph limit $\Gamma$ if and only if $\left|V\left(G_{n}\right)\right| \rightarrow \infty$ and $p\left(H ; G_{n}\right) \rightarrow p(H ; \Gamma)$ for every graph $H$. Moreover, a graph limit is uniquely determined by the numbers $p(H ; \Gamma)$ for all $H \in \mathcal{U}$.

It is shown in LS06 that every graph limit $\Gamma$ can be represented by a graphon, which is a symmetric measurable function $W:[0,1]^{2} \rightarrow[0,1]$. The set of all graphons are denoted by $\mathcal{W}_{0}$. Given a graph $G$ with vertex set $[n]$, we define the corresponding graphon $W_{G}:[0,1]^{2} \rightarrow\{0,1\}$ as follows. Let $W_{G}(x, y):=$ $A_{G}(\lceil x n\rceil,\lceil y n\rceil)$ if $x, y \in(0,1]$, and if $x=0$ or $y=0$, set $W_{G}$ to 0 . It is easy to see that if $\left(G_{n}\right)_{n=1}^{\infty}$ is a graph sequence that converges to a graph limit $\Gamma$, then for every graph $H$,

$$
p(H ; \Gamma)=\lim _{n \rightarrow \infty} \mathbb{E}\left[\prod_{u v \in E(H)} W_{G_{n}}\left(x_{u}, x_{v}\right) \prod_{u v \in E(H)^{c}}\left(1-W_{G_{n}}\left(x_{u}, x_{v}\right)\right)\right],
$$

where $\left\{x_{u}\right\}_{u \in V(H)}$ are independent random variables taking values in $[0,1]$ uniformly, and $E(H)^{c}=\{u v$ : $u \neq v, u v \notin E(H)\}$. Lovász and Szegedy [LS06] showed that for every graph limit $\Gamma$, there exists a graphon 
$W$ such that for every graph $H$, we have $p(H ; \Gamma)=p(H ; W)$ where

$$
p(H ; W):=\mathbb{E}\left[\prod_{u v \in E(H)} W\left(x_{u}, x_{v}\right) \prod_{u v \in E(H)^{c}}\left(1-W\left(x_{u}, x_{v}\right)\right)\right] .
$$

Furthermore, this graphon is unique in the following sense: If $W_{1}$ and $W_{2}$ are two different graphons representing the same graph limit, then there exists a measure-preserving map $\sigma:[0,1] \rightarrow[0,1]$ such that

$$
W_{1}(x, y)=W_{2}(\sigma(x), \sigma(y))
$$

almost everywhere [BCL10]. With these considerations, sometimes we shall not distinguish between the graph limits and their corresponding graphons. We define the $\delta_{1}$ distance of two graphons $W_{1}$ and $W_{2}$ as

$$
\delta_{1}\left(W_{1}, W_{2}\right)=\inf \left\|W_{1}-W_{2} \circ \sigma\right\|_{1},
$$

where the infimum is over all measure-preserving maps $\sigma:[0,1] \rightarrow[0,1]$.

A graphon $W$ is called a stepfunction, if there is a partition of $[0,1]$ into a finite number of measurable sets $S_{1}, \ldots, S_{n}$ so that $W$ is constant on every $S_{i} \times S_{j}$. The partition classes will be called the steps of $W$.

Let $W$ be a graphon and $x_{1}, \ldots, x_{n} \in[0,1]$. The random graph $G\left(x_{1}, \ldots, x_{n}, W\right) \in \mathcal{L}_{n}$ is obtained by including the edge $i j$ with probability $W\left(x_{i}, x_{j}\right)$, independently for all pairs $(i, j)$ with $1 \leq i<j \leq n$. By picking $x_{1}, \ldots, x_{n}$ independently and uniformly at random from $[0,1]$, we obtain the random graph $G(n, W) \in \mathcal{L}_{n}$. Note that that for every $H \in \mathcal{L}_{n}$,

$$
\operatorname{Pr}[G(n, W)=H]=p(H ; W) .
$$

1.2. Graph properties and Entropy. A subset of the set $\mathcal{U}$ is called a graph class. Similarly a graph property is a property of graphs that is invariant under graph isomorphisms. There is an obvious one-to-one correspondence between graph classes and graph properties and we will not distinguish between a graph property and the corresponding class. Let $\mathcal{Q} \subseteq \mathcal{U}$ be a graph class. For every $n>1$, we denote by $\mathcal{Q}_{n}$ the set of graphs in $\mathcal{Q}$ with exactly $n$ vertices. We let $\overline{\mathcal{Q}} \subseteq \overline{\mathcal{U}}$ be the closure of $\mathcal{Q}$ in $\overline{\mathcal{U}}$.

Define the binary entropy function $h:[0,1] \mapsto \mathbb{R}_{+}$as $h(x)=-x \log (x)-(1-x) \log (1-x)$ for $x \in(0,1)$ and $h(0)=h(1)=0$ so that $h$ is continuous on $[0,1]$ where here and throughout the paper $\log (\cdot) \operatorname{denotes}$ the logarithm to the base 2 . The entropy of a graphon $W$ is defined as

$$
\operatorname{Ent}(W):=\int_{0}^{1} \int_{0}^{1} h(W(x, y)) d x d y .
$$

Note that it follows from the uniqueness result (1.1) that entropy is a function of the underling graph limit, and it does not depend on the choice of the graphon representing it. It is shown in Ald85, and Jan, Theorem D.5] that

$$
\lim _{n \rightarrow \infty} \frac{\operatorname{Ent}(G(n, W))}{\left(\begin{array}{l}
n \\
2
\end{array}\right)}=\operatorname{Ent}(W)
$$

A graphon is called random-free if it is $\{0,1\}$-valued almost everywhere. Note that a graphon $W$ is randomfree if and only if $\operatorname{Ent}(W)=0$, which by (1.2) is equivalent to $\operatorname{Ent}(G(n, W))=o\left(n^{2}\right)$. Our first theorem shows that this is sharp in the sense that the growth of $\operatorname{Ent}(G(n, W))$ for random-free graphons $W$ can be arbitrarily close to quadratic.

Theorem 1.1. Let $\alpha: \mathbb{N} \rightarrow \mathbb{R}_{+}$be a function with $\lim _{n \rightarrow \infty} \alpha(n)=0$. Then there exists a random-free graphon $W$ such that $\operatorname{Ent}(G(n, W))=\Omega\left(\alpha(n) n^{2}\right)$.

A graph property $\mathcal{Q}$ is called random-free if every $W \in \overline{\mathcal{Q}}$ is random-free. Our next theorem shows that in contrast to Theorem 1.1 when a graphon $W$ is the limit of a sequence of graphs with a random-free property, then $\operatorname{Ent}(G(n, W))$ cannot grow faster than $O(n \log n)$.

Theorem 1.2. Let $\mathcal{Q}$ be a random-free property, and let $W$ be the limit of a sequence of graphs in $\mathcal{Q}$. Then $\operatorname{Ent}(G(n, W))=O(n \log n)$. 
Remark 1.3. We defined $G(n, W)$ as a labeled graph in $\mathcal{L}_{n}$. Both Theorems 1.1 and 1.2 remain valid if we consider the random variable $G_{u}(n, W)$ taking values in $\mathcal{U}_{n}$ obtained from $G(n, W)$ by forgetting the labels. Indeed, $\operatorname{Ent}\left(G_{u}(n, W)\right)=\operatorname{Ent}(G(n, W))-\operatorname{Ent}\left(G(n, W) \mid G_{u}(n, W)\right)$ and $\operatorname{Ent}\left(G(n, W) \mid G_{u}(n, W)=H\right)=$ $O(n \log n)$ for every $H \in \mathcal{U}_{n}$. It follows that

$$
\operatorname{Ent}(G(n, W))-O(n \log n) \leq \operatorname{Ent}\left(G_{u}(n, W)\right) \leq \operatorname{Ent}(G(n, W)) .
$$

\section{Proof of Theorem 1.1}

For every positive integer $m$, let $F_{m}$ denote the unique bigraph $\left([m],\left[2^{m}\right], E\right)$ with the property that the vertices in $\left[2^{m}\right]$ all have different sets of neighbors. The transversal-uniform graph is the unique graph (up to an isomorphism) with vertex set $\mathbb{N}$ which satisfies the following property. The vertices are partitioned into sets $\left\{A_{i}\right\}_{i=1}^{\infty}$ with $\log \left|A_{i}\right|=\sum_{j=1}^{i-1}\left|A_{i-1}\right|$. There are no edges inside $A_{i}$ 's, and for every $i$, the bigraph induced by $\left(\cup_{j=1}^{i-1} A_{j}, A_{i}\right)$ is isomorphic to $F_{\sum_{j=1}^{i-1}\left|A_{j}\right|}$.

Let $\mathcal{I}=\left\{I_{i}\right\}_{i \in \mathbb{N}}$ be a partition of $[0,1]$ into intervals. We define its corresponding transversal-uniform graphon $W_{\mathcal{I}}$ by assigning weights $\left|I_{i}\right| /\left|A_{i}\right|$ to all the vertices in $A_{i}$ in the transversal-uniform graph $G_{U}$ described above. More precisely, we partition each $I_{i}$ into $\left|A_{i}\right|$ equal size intervals (corresponded with elements in $A_{i}$ ), and mapping all the points in each of these subintervals to its corresponding vertex in $A_{i}$. This measurable surjection $\pi_{\mathcal{I}}:[0,1] \rightarrow \mathbb{N}$, together with the transversal-uniform graph described above defines the transversal-uniform graphon $W_{\mathcal{I}}$ by setting

$$
W_{\mathcal{I}}(x, y)= \begin{cases}1 & \text { if } \pi(x) \pi(y) \in E\left(G_{U}\right), \\ 0 & \text { if } \pi(x) \pi(y) \notin E\left(G_{U}\right) .\end{cases}
$$

Note that by construction $W_{\mathcal{I}}$ has the following property. Let $s<k$ be positive integers, and $x_{1}, \ldots, x_{s} \in$ $\cup_{i<k} I_{i}$ belong to pairwise distinct intervals in $\mathcal{I}$. For every $f:[s] \rightarrow\{0,1\}$, we have

$$
\operatorname{Pr}\left[\forall i, W_{\mathcal{I}}\left(x_{i}, y\right)=f(i) \mid y \in I_{k}\right]=\frac{1}{2^{s}},
$$

where $y$ is a random variable taking values uniformly in $[0,1]$. It follows that for every graph $H$ on $s$ vertices,

$$
\operatorname{Pr}\left[G\left(x_{1}, \ldots, x_{s}, W_{\mathcal{I}}\right)=H \mid \forall i, x_{i} \in I_{k_{i}}\right]=\frac{1}{2^{\left(\begin{array}{c}
s \\
2
\end{array}\right)}},
$$

where $x_{1}, \ldots, x_{s}$ are now i.i.d. random variables taking values uniformly in $[0,1]$, and $k_{1}, k_{2}, \ldots, k_{s}$ are distinct natural numbers.

We translate (2.1) into a lower bound on (conditional) entropy of transversal-uniform graphons. First we need a simple lemma.

Lemma 2.1. Let $W_{\mathcal{I}}$ be a transversal-uniform graphon, and $\phi:[n] \rightarrow[0,1]$ be a uniformly random map. For every $\rho:[n] \rightarrow \mathbb{N}$, we have

$$
\operatorname{Ent}\left(G\left(\phi(1), \ldots, \phi(n), W_{\mathcal{I}}\right) \mid \pi_{\mathcal{I}} \circ \phi=\rho\right) \geq\left(\begin{array}{c}
|\operatorname{Im}(\rho)| \\
2
\end{array}\right) .
$$

Proof. Pick a set of representatives $K \subseteq[n]$ so that $\left.\rho\right|_{K}: K \rightarrow \operatorname{Im}(\rho)$ is a bijection. Equation (2.1) implies that for every graph $H$ with $V(H)=K$,

$$
\operatorname{Pr}\left[G\left(\phi(1), \ldots, \phi(n), W_{\mathcal{I}}\right)[K]=H \mid \pi_{\mathcal{I}} \circ \phi=\rho\right]=\frac{1}{2^{\left({ }_{2}^{|\operatorname{Im}(\rho)|}\right)}} .
$$

Therefore,

$$
\operatorname{Ent}\left(G\left(\phi(1), \ldots, \phi(n), W_{\mathcal{I}}\right) \mid \pi_{\mathcal{I}} \circ \phi=\rho\right) \geq \operatorname{Ent}\left(G\left(\phi(1), \ldots, \phi(n), W_{\mathcal{I}}\right)[K] \mid \pi_{\mathcal{I}} \circ \phi=\rho\right)=\left(\begin{array}{c}
|\operatorname{Im}(\rho)| \\
2
\end{array}\right) .
$$


In the proof of Theorem 1.1 below we will make use of the following well-known inequality about conditional entropy. For discrete random variables $X$ and $Y$,

$$
\operatorname{Ent}(X \mid Y):=\sum_{y \in \operatorname{supp}(Y)} \operatorname{Pr}[Y=y] \operatorname{Ent}(X \mid Y=y) \leq \operatorname{Ent}(X) .
$$

Proof of Theorem 1.1. For every positive integer $k$, define

$$
g_{k}:=\max \left\{\left\{2^{k+5}\right\} \cup\left\{n \mid \alpha(n)>2^{-2 k-9}\right\}\right\} .
$$

The numbers $g_{k}$ are well-defined, as the condition $\lim _{n \rightarrow \infty} \alpha(n)=0$ implies that the set $\left\{n \mid \alpha(n)>2^{-2 k-9}\right\}$ is finite. Define the sums $G_{k}:=\sum_{i=1}^{k} g_{k}$, and set $\beta_{i}=\frac{1}{g_{k} 2^{k}}$ for all the $g_{k}$ indices $i \in\left(G_{k-1}, G_{k}\right]$. Let $\mathcal{I}=\left\{I_{i}\right\}_{i \in \mathbb{N}}$ be a partition of $[0,1]$ into intervals with $\left|I_{i}\right|=\beta_{i}$, and let $W_{\mathcal{I}}$ be the corresponding transversaluniform graphon.

Consider a sufficiently large $n \in \mathbb{N}$, and let $k \in \mathbb{N}$ be chosen to be maximum so that $2^{k+4} \leq n$ and $\alpha(n) \leq 2^{-2 k-7}$. We have $n<2^{k+5}$ or $\alpha(n)>2^{-2 k-9}$. Therefore $n \leq g_{k}$ by the definition of $g_{k}$. Let $\phi:[n] \rightarrow[0,1]$ be random and uniform. By Lemma 2.1 for any fixed $\rho:[n] \rightarrow \mathbb{N}$, we have

$$
\operatorname{Ent}\left(G\left(\phi(1), \ldots, \phi(n), W_{\mathcal{I}}\right) \mid \pi_{\mathcal{I}} \circ \phi=\rho\right) \geq\left(\begin{array}{c}
|\operatorname{Im}(\rho)| \\
2
\end{array}\right) .
$$

Thus

$$
\operatorname{Ent}\left(G\left(n, W_{\mathcal{I}}\right)\right) \geq \operatorname{Ent}\left(G\left(n, W_{\mathcal{I}}\right) \mid \pi_{\mathcal{I}} \circ \phi\right) \geq \operatorname{Pr}\left[\left|\operatorname{Im}\left(\pi_{\mathcal{I}} \circ \phi\right)\right| \geq n 2^{-k-2}\right]\left(\begin{array}{c}
n 2^{-k-2} \\
2
\end{array}\right) .
$$

Define the random variable $X:=\left|\operatorname{Im}\left(\pi_{\mathcal{I}} \circ \phi\right) \cap\left(G_{k-1}, G_{k}\right]\right| \leq\left|\operatorname{Im}\left(\pi_{\mathcal{I}} \circ \phi\right)\right|$. We have

$$
\mathbb{E}[X]=\sum_{i \in\left(G_{k-1}, G_{k}\right]} \operatorname{Pr}\left[\phi^{-1}\left(I_{i}\right) \neq \emptyset\right]=\sum_{i \in\left(G_{k-1}, G_{k}\right]}\left(1-\left(1-\beta_{i}\right)^{n}\right)=g_{k}\left(1-\left(1-\frac{1}{g_{k} 2^{k}}\right)^{n}\right) \geq n 2^{-k-1},
$$

where we used the fact that $g_{k} 2^{k} \geq 2 n$ and that $(1-x)^{n} \leq 1-n x+n^{2} x^{2} \leq 1-n x / 2$ for $x \in[0,1 / 2 n]$. As the events $\phi^{-1}\left(I_{i}\right) \neq \emptyset$ and $\phi^{-1}\left(I_{j}\right) \neq \emptyset$ are negatively correlated for $i \neq j$, we have $\operatorname{Var}[X] \leq \mathbb{E}[X]$. Hence by Chebyshev's inequality

$$
\begin{aligned}
\operatorname{Pr}\left[\left|\operatorname{Im}\left(\pi_{\mathcal{I}} \circ \phi\right)\right| \geq n 2^{-k-2}\right] & \geq \operatorname{Pr}\left[X \geq n 2^{-k-2}\right] \geq 1-\operatorname{Pr}\left[|X-\mathbb{E}[X]| \geq \frac{\mathbb{E}[X]}{2}\right] \\
& \geq 1-\frac{4 \operatorname{Var}[X]}{\mathbb{E}[X]^{2}} \geq 1-\frac{4}{n 2^{-k-2}} \geq \frac{1}{2} .
\end{aligned}
$$

Substituting in (2.3) we obtain

$$
\operatorname{Ent}\left(G\left(n, W_{\mathcal{I}}\right)\right) \geq \frac{1}{2}\left(\begin{array}{c}
n 2^{-k-2} \\
2
\end{array}\right) \geq n^{2} 2^{-2 k-7} \geq \alpha(n) n^{2},
$$

as desired.

\section{Proof of Theorem 1.2}

In [LS] Lovász and Szegedy obtained a combinatorial characterization of random-free graph properties. To state this result it is convenient to distinguish between bipartite graphs and bigraphs. A bipartite graph is a graph $(V, E)$ whose node set has a partition into two classes such that all edges connect nodes in different classes. A bigraph is a triple $\left(U_{1}, U_{2}, E\right)$ where $U_{1}$ and $U_{2}$ are finite sets and $E \subseteq U_{1} \times U_{2}$. So a bipartite graph becomes a bigraph if we fix a bipartition and specify which bipartition class is first and second. On the other hand, if $F=(V, E)$ is a graph, then $\left(V, V, E^{\prime}\right)$ is an associated bigraph, where $E^{\prime}=\{(x, y): x y \in E\}$.

If $G=(V, E)$ is a graph, then an induced sub-bigraph of $G$ is determined by two (not necessarily disjoin) subsets $S, T \subseteq V$, and its edge set consists of those pairs $(x, y) \in S \times T$ for which $x y \in E$ (so this is an induced subgraph of the bigraph associated with $G$ ). 
For a bigraph $H=\left(U_{1}, U_{2}, E\right)$ and a graphon $W$, analogous to the definition of the induced density of a graph in a graphon, we define

$$
p^{\mathrm{b}}(H ; W)=\mathbb{E}\left[\prod_{\substack{u \in U_{1}, v \in U_{2} \\ u v \in E}} W\left(x_{u}, y_{v}\right) \prod_{\substack{u \in U_{1}, v \in U_{2} \\ u v \in\left(U_{1} \times U_{2}\right) \backslash E}}\left(1-W\left(x_{u}, y_{v}\right)\right)\right],
$$

where $\left\{x_{u}\right\}_{u \in U_{1}},\left\{y_{v}\right\}_{v \in U_{2}}$ are independent random variables taking values in [0,1] uniformly. Now we are ready to state Lovász and Szegedy's characterization of random-free graph properties.

Theorem 3.1. [LS] A graph property $\mathcal{Q}$ is random-free if and only if there exists a bigraph $H$ such that $p^{\mathrm{b}}(H ; W)=0$ for all $W \in \overline{\mathcal{Q}}$.

The following lemma is due to Alon, Fischer, and Newman (See AFN07, Lemma 1.6]).

Lemma 3.2. AFN07 Let $k$ be a fixed integer and let $\delta>0$ be a small real. For every graph $G$, either there exists stepfunction graphon $W^{\prime}$ with $r \leq\left(\frac{k}{\delta}\right)^{O(k)}$ steps such that $\delta_{1}\left(W_{G}, W^{\prime}\right) \leq \delta$, or for every bigraph $H$ on $k$ vertices $p^{b}(H ; G) \geq\left(\frac{\delta}{k}\right)^{O\left(k^{2}\right)}$.

Every random-free graphon $W$ can be approximated arbitrarily well in the $\delta_{1}$ distance with $W_{G}$ for some graph $G$, and furthermore, for every fixed $H$, the function $p^{\mathrm{b}}(H, \cdot)$ is continuous in the $\delta_{1}$ distance. Thus Lemma 3.2 can be generalized to random-free graphons.

Corollary 3.3. Let $k$ be a fixed integer and let $\delta>0$ be a small real. For every random-free graphon $W$, either there exists a stepfunction graphon $W^{\prime}$ with $r \leq\left(\frac{k}{\delta}\right)^{O(k)}$ steps such that $\delta_{1}\left(W, W^{\prime}\right) \leq \delta$, or for every bigraph $H$ on $k$ vertices $p^{b}(H ; G) \geq\left(\frac{\delta}{k}\right)^{O\left(k^{2}\right)}$.

Next we will prove two simple lemmas about entropy.

Lemma 3.4. Let $\mu_{1}$ and $\mu_{2}$ be two discrete probabilistic distributions on a finite set $\Omega$. Then

$$
\left|\operatorname{Ent}\left(\mu_{1}\right)-\operatorname{Ent}\left(\mu_{2}\right)\right| \leq|\Omega| h\left(\frac{\left\|\mu_{1}-\mu_{2}\right\|_{1}}{|\Omega|}\right) .
$$

Proof. Define $0 \log 0:=\lim _{x \rightarrow 0} x \log x=0$. By taking the derivative with respect to $x$, for fixed $d$ we see that $(x+d) \log (x+d)-x \log x$ is monotone for $0 \leq x \leq 1-d$. Therefore, for $x_{1}, x_{2} \in[0,1]$ we have

$$
\left|x_{2} \log x_{2}-x_{1} \log x_{1}\right| \leq \max \left\{-\left|x_{2}-x_{1}\right| \log \left|x_{2}-x_{1}\right|,-\left(1-\left|x_{2}-x_{1}\right|\right) \log \left(1-\left|x_{2}-x_{1}\right|\right)\right\} \leq h\left(\left|x_{2}-x_{1}\right|\right) .
$$

Thus

$$
\begin{aligned}
\left|\operatorname{Ent}\left(\mu_{1}\right)-\operatorname{Ent}\left(\mu_{2}\right)\right| & =\left|\sum_{x \in \Omega} \mu_{1}(x) \log \mu_{1}(x)-\mu_{2}(x) \log \mu_{2}(x)\right| \\
& \leq \sum_{x \in \Omega} h\left(\left|\mu_{1}(x)-\mu_{2}(x)\right|\right) \leq|\Omega| h\left(\frac{\left\|\mu_{1}-\mu_{2}\right\|_{1}}{|\Omega|}\right),
\end{aligned}
$$

where the last inequality is by concavity of the binary entropy function $h$.

Lemma 3.5. Let $W_{1}$ and $W_{2}$ be two graphons, and let $\mu_{1}$ and $\mu_{2}$ be the probability distributions on $\mathcal{L}_{n}$ induced by $G\left(n, W_{1}\right)$ and $G\left(n, W_{2}\right)$, respectively. Then

$$
\left\|\mu_{1}-\mu_{2}\right\|_{1} \leq n^{2} \delta_{1}\left(W_{1}, W_{2}\right) .
$$

Proof. Let $x_{1}, \ldots, x_{n}$ be i.i.d. uniform random variables with values in $[0,1]$. Note

$$
\begin{aligned}
\left\|\mu_{1}-\mu_{2}\right\|_{1} & \leq \operatorname{Pr}\left[G\left(x_{1}, \ldots, x_{n}, W_{1}\right) \neq G\left(x_{1}, \ldots, x_{n}, W_{2}\right)\right] \\
& \leq \mathbb{E}\left[\sum_{i \neq j} \mid W_{1}\left(x_{i}, x_{j}\right)-W_{2}\left(x_{i}, x_{j}\right)\right] \leq n^{2}\left\|W_{1}-W_{2}\right\|_{1} .
\end{aligned}
$$


Proof of Theorem 1.Q. Since $\mathcal{Q}$ is random-free, by Theorem 3.1] there exists a bigraph $H$ such that $p^{\mathrm{b}}(H ; W)=$ 0 for all $W \in \overline{\mathcal{Q}}$. Applying Corollary 3.3 with $\delta=1 / n^{5}$ shows that there exists a stepfunction graphon $W^{\prime}$ with $n^{O(1)}$ steps satisfying $\left\|W-W^{\prime}\right\|_{1} \leq \delta$. Then since $\left|\mathcal{L}_{n}\right| \leq 2^{n^{2}}$, Lemmas 3.4 and 3.5 imply

$$
\begin{aligned}
\mid \operatorname{Ent}\left(G\left(n, W^{\prime}\right)\right) & -\operatorname{Ent}(G(n, W)) \mid \leq 2^{n^{2}} h\left(\frac{n^{2} \delta}{2^{n^{2}}}\right) \\
& =-2^{n^{2}}\left(\frac{n^{2} \delta}{2^{n^{2}}} \log \left(\frac{n^{2} \delta}{2^{n^{2}}}\right)+\left(1-\frac{n^{2} \delta}{2^{n^{2}}}\right) \log \left(1-\frac{n^{2} \delta}{2^{n^{2}}}\right)\right) \\
& \leq n^{4} \delta+n^{2} \delta(-2 \log n-\log \delta)+2^{n^{2}} \cdot 2 \frac{n^{2} \delta}{2^{n^{2}}}=o(1) .
\end{aligned}
$$

Since $W^{\prime}$ is random-free and it has $n^{O(1)}$ steps, $\left|\operatorname{supp}\left(G\left(n, W^{\prime}\right)\right)\right|=n^{O(n)}$. Consequently $\operatorname{Ent}\left(G\left(n, W^{\prime}\right)\right)=$ $O(n \log n)$.

\section{Concluding Remarks}

1. Note that if $W$ is a random-free stepfunction, then $\operatorname{Ent}(G(n, W))=O(n)$. In Jan it is conjectured that the converse is also true. That is $\operatorname{Ent}(G(n, W))=O(n)$ if and only if $W$ is equivalent to a random-free stepfunction. The following simple example disproves this conjecture.

Let $\mu$ be the probability distribution on $\mathbb{N}$ defined by $\mu(\{i\})=2^{-i}$. Consider the random variable $X=\left(X_{1}, \ldots, X_{n}\right) \in \mathbb{N}^{n}$ where $X_{i}$ are i.i.d. random variables with distribution $\mu$. We have $\operatorname{Ent}\left(X_{i}\right)=$ $\sum_{i=1}^{\infty} 2^{-i} i=2$. Hence $\operatorname{Ent}(X) \leq \sum \operatorname{Ent}\left(X_{i}\right)=2 n$.

Partition $[0,1]$ into intervals $\left\{I_{i}\right\}_{i=1}^{\infty}$ where $\left|I_{i}\right|=2^{-i}$. Let $W$ be the graphon that is constant 1 on $\cup_{i=1}^{\infty} I_{i} \times I_{i}$ and 0 everywhere else. Note that

$$
\operatorname{Ent}(G(n, W)) \leq \operatorname{Ent}(X) \leq 2 n .
$$

Therefore $G(n, W)$ has linear entropy.

It remains to verify that $W$ is not equivalent to a stepfunction. This follows immediately from the fact that $W$ has infinite rank as a kernel. It can also be verified in a more combinatorial way: A homogenous set of vertices in a graph $H$ is a set of vertices which are either all pairwise adjacent to each other, or all pairwise non-adjacent. If $W$ is equivalent to a step-function with $k$ steps, then every $H \in \operatorname{supp}(G(n, W))$ cleary contains a homogenous set of size at least $n / k$. On the other hand, if $H \in \mathcal{L}_{n^{2}}$ is a disjoint union of $n$ complete graphs on $n$ vertices, then the largest homogenous set in $H$ has size $n$, but $H \in \operatorname{supp}\left(G\left(n^{2}, W\right)\right)$ by construction.

2. Theorem 1.2 shows that when $W$ is a limit of a random-free property, then the entropy of $G(n, W)$ is small. However, the support of $G(n, W)$ can be comparatively large. For every $\epsilon>0$, we construct examples for which $\log (|\operatorname{supp}(G(n, W))|)=\Omega\left(n^{2-\epsilon}\right)$. Note that Theorem 1.2 implies that $G(n, W)$ is far from being uniform on the support in these examples, as the entropy of a uniform random variable with support of size $2^{\Omega\left(n^{2-\epsilon}\right)}$ is $\Omega\left(n^{2-\epsilon}\right)$.

Let us now describe the construction. Let $\mathcal{Q}$ be the set of graphs that do not contain $K_{t, t}$ as a subgraph. Partition $[0,1]$ into intervals $\left\{S_{i}\right\}_{i=1}^{\infty}$ with non-zero lengths, and let $\left\{H_{i}\right\}_{i=1}^{\infty}$ be an enumeration of graphs in $\mathcal{Q}$. Define $W$ to be the graphon that is 0 on $S_{i} \times S_{j}$ for $i \neq j$, and is equivalent to $W_{H_{i}}$ (scaled properly) on $S_{i} \times S_{i}$. By construction $p(H ; W)>0$ if $H \in \mathcal{Q}$. Thus $|\operatorname{supp}(G(n, W))| \geq\left|\mathcal{Q}_{n}\right|$. Since there exists $K_{t, t}$-free graphs with $n^{2-2 / t}$ edges (See e.g. [Bol78, p. 316, Thm. VI.2.10]), we have $\left|\mathcal{Q}_{n}\right| \geq 2^{n^{2-2 / t}}$.

It remains to show that $W$ is a limit of graphs in some random-free property. Unfortunately, $W \notin \overline{\mathcal{Q}}$. We construct a larger random-free property $\mathcal{Q}^{\prime}$ so that $W \in \overline{\mathcal{Q}^{\prime}}$ as follows.

Fix a bigraph $B$, so that the corresponding graph contains $K_{t, t}$ as a subgraph and is connected. Suppose further that no two vertices of $B$ have the same neighborhood. Note that such a bigraph trivially exists. For example, one can take $B=\left(V_{1} \cup U_{1}, V_{2} \cup U_{2}, E\right)$ so that $V_{1}, U_{1}, V_{2}, U_{2}$ are disjoint sets of size $t$, every vertex of $V_{1}$ is joined to every vertex of $V_{2}$, and the edges between $V_{1}$ and $U_{2}$, as well as the edges between $U_{1}$ and $V_{2}$, form a matching of size $t$. Let $\mathcal{Q}^{\prime} \supseteq \mathcal{Q}$ be the set of graphs not containing $B$ as an induced sub-bigraph. Then $\mathcal{Q}^{\prime}$ is random-free by Theorem 3.1, as $p^{\mathrm{b}}\left(B, W^{\prime}\right)=0$ for every $W^{\prime} \in \overline{\mathcal{Q}^{\prime}}$. 
Let $r=|V(B)|$ and suppose that $G=G\left(x_{1}, x_{2}, \ldots, x_{r}, W\right)$ contains $B$ as an induced sub-bigraph. Then there exists $i$ so that $x_{1}, x_{2}, \ldots, x_{r} \in S_{i}$, as $G$ is connected. It follows further that $G$ is an induced subgraph of $H_{i}$, as no two vertices of $G$ have the same neighborhood. Thus $G$ contains no $K_{t, t}$ subgraph, contradicting our assumption that $G$ contains $B$. We conclude that $\operatorname{supp}(G(n, W)) \subseteq \mathcal{Q}^{\prime}$ for every positive integer $n$. By [LS06, Lemma 2.6] the sequence $\{G(n, W)\}_{n=1}^{\infty}$ converges to $W$ with probability one. Thus $W \in \overline{\mathcal{Q}^{\prime}}$, as desired.

\section{REFERENCES}

[AFN07] Noga Alon, Eldar Fischer, and Ilan Newman. Efficient testing of bipartite graphs for forbidden induced subgraphs. SIAM J. Comput., 37(3):959-976 (electronic), 2007.

[Ald85] David J. Aldous. Exchangeability and related topics. In École d'été de probabilités de Saint-Flour, XIII-1983, volume 1117 of Lecture Notes in Math., pages 1-198. Springer, Berlin, 1985.

[BCL10] Christian Borgs, Jennifer Chayes, and László Lovász. Moments of two-variable functions and the uniqueness of graph limits. Geom. Funct. Anal., 19(6):1597-1619, 2010.

[Bol78] Béla Bollobás. Extremal graph theory, volume 11 of London Mathematical Society Monographs. Academic Press Inc. [Harcourt Brace Jovanovich Publishers], London, 1978.

[HJS] Hamed Hatami, Svante Janson, and Balázs Szegedy. in preparation.

[Jan] Svante Janson. Graphons, cut norm and distance, couplings and rearrangements. ArXiv:1009.2376.

[LS] László Lovász and Balázs Szegedy. Regularity partitions and the topology of graphons. arXiv:1002.4377.

[LS06] László Lovász and Balázs Szegedy. Limits of dense graph sequences. J. Combin. Theory Ser. B, 96(6):933-957, 2006.

School of Computer Science, McGill University, Montreal, Canada.

E-mail address: hatami@cs.mcgill.ca

Department of Mathematics \& Statistics, McGill University, Montreal, Canada.

E-mail address: snorin@math.mcgill.ca 